\title{
Global Periodic Solutions of the Nonlinear Wave Equation
}

\author{
Lamberto Cesari \& Patrizia Pucci
}

\author{
Communicated by J. SERRIN
}

\section{Introduction}

We consider here the problem

$$
\begin{aligned}
u_{t t}-u_{r \tau} & =\phi(t, \tau)+g(u), \quad(t, \tau) \in \mathbb{R}^{2}, \\
u(t+T, \tau) & =u(t, \tau)=u(t, \tau+T), \\
\phi(t+T, \tau) & =\phi(t, \tau)=\phi(t, \tau+T),
\end{aligned}
$$

where $u$ and $\phi$ denote scalar functions on $\mathbb{R}^{2}$, and $g$ is a scalar function on $\mathbb{R}$. We shall assume $g$ and $\phi$ continuous, and we shall determine sufficient conditions in the form of inequalities to guarantee that problem (1) has continuous solutions. Thus, the solutions which we consider will be weak solutions in the sense of distributions.

In terms of functional analysis and the alternative approach [cf. 3, 6], problem (1) is difficult since the kernel of the underlying operator $\Xi u=u_{t t}-u_{\tau \tau}$, with $u$ doubly $T$-periodic, is infinite dimensional.

Cesari [1], Hale [5], and more recently Cesari and KanNan [4] have considered problem (1) and the analogous problem

$$
\left\{\begin{aligned}
U_{x y} & =\Phi(x, y)+G(U), \quad(x, y) \in R^{2}, \\
U(x+T, y) & =U(x, y)=U(x, y+T), \\
\Phi(x+T, y) & =\Phi(x, y)=\Phi(x, y+T),
\end{aligned}\right.
$$

in different settings and by a uniform alternative approach. Though the usual transformation, $x=t+\tau, \quad y=t-\tau$, takes the linear operator $u_{t t}-u_{t \tau}$ into the linear operator $U_{x y}$, the boundary conditions do not match.

In the present paper the relations between problems (1) and (2) are closely investigated in terms of functional analysis and suitable projection operators. Then problem (1) is discussed by an alternative approach, yielding sufficient conditions for the existence of continuous solutions. 
For elliptic problems LANDESMAN and LAZER showed, again by an alternative approach, that qualitative condition on $g$ suffice. For instance, the condition

$$
g(x) \geqq B \text { for } x \geqq b ; \quad g(x) \leqq-B \text { for } x \leqq-b,
$$

is sufficient to guarantee the existence of solutions of $\Xi u=\Phi(t, \tau)+g(u)$, $(t, \tau) \in \Omega, u=0$ on $\partial \Omega$, where $\Xi$ is a strongly uniformly elliptic operator, $\Phi \in L_{2}(\Omega)$, and $|\Phi|$ is "not too large" (cf. [3]).

For hyperbolic problems such as (1) and (2) it appears that this is not the case. We shall use the same condition (3), but together with a number of quantitative restrictions in the form of algebraic inequalities. The analogous situation is wellknown in other global existence theorems for hyperbolic problems (e.g., L. CESARI [2], P. PUCCI [7]), where existence has also been proved under a set of restrictions in the form of algebraic inequalities. At the same time, existence theorems for problem (1) under qualitative assumtions are also known for globally monotone functions $g$. We have tried not to use this condition here.

\section{Reduction of the Wave Equation in $\mathbf{R}^{2}$ to the Canonical Equation}

For a given periodic function $f: \mathbb{R}^{2} \rightarrow \mathbb{R}$ of period $T$ in $t$ and $\tau$ (briefly, doubly $T$-periodic), we consider the problem of doubly $T$-periodic solutions $u=u(t, \tau)$ of the linear wave equation

$$
\begin{array}{r}
u_{t t}-u_{\tau \tau}=f(t, \tau), \quad(t, \tau) \in \mathbb{R}^{2}, \\
u(t+T, \tau)=u(t, \tau)=u(t, \tau+T), \\
f(t+T, \tau)=f(t, \tau)=f(t, \tau+T),
\end{array}
$$

Let us consider the relations

$$
t+\tau=x, \quad t-\tau=y ; \quad t=(x+y) / 2, \quad \tau=(x-y) / 2 .
$$

We denote by $A$ the operation $(t, \tau) \rightarrow(x, y)$ defined by these relations, and by $A^{-1}$ the inverse operation $(x, y) \rightarrow(t, \tau)$. Then the differential equation in (1) is reduced to the form

$$
U_{x y}=F(x, y)
$$

with

$$
\begin{array}{cl}
U(x, y)=u((x+y) / 2,(x-y) / 2), & F(x, y)=\frac{1}{4} f((x+y) / 2,(x-y) / 2), \\
u(t, \tau)=U(t+\tau, t-\tau), & f(t, \tau)=4 F(t+\tau, t-\tau),
\end{array}
$$

and now $F$ is doubly $2 T$-periodic.

Even if we were able to determine doubly $2 T$-periodic solutions $U$ of (2), the corresponding functions $u$ would not necessarily be doubly $T$-periodic. For instance, for $T=2 \pi, u_{t t}-u_{\tau \tau}=\sin t$ becomes $U_{x y}=\frac{1}{4} \sin (x+y) / 2$, which has the doubly $4 \pi$-periodic solution $U(x, y)=-\sin (x+y) / 2+\sin x / 2$. The 
corresponding function $u(t, \tau)=-\sin t+\sin (t+\tau) / 2$ is a doubly $4 \pi$-periodic solution of (1), not a doubly $2 \pi$-periodic one.

We shall first describe (see CeSARI [1]) the alternative process for the determination of doubly $2 T$-periodic solutions of (2), and then we shall discuss a modification of the process to obtain doubly $T$-periodic solutions of (1).

\section{An Alternative Process for the Doubly 2T-Periodic Solutions of the Equation $U_{x y}=F$}

Let $F(x, y)$, or $F: \mathbb{R}^{2} \rightarrow \mathbb{R}$, be a given doubly $2 T$-periodic function, which we shall assume to be continuous in $\mathbb{R}^{2}$. Let the functions $m(y), n(x)$, or $m, n: \mathbb{R} \rightarrow \mathbb{R}$, and the constant $\mu$ be defined by

$$
\begin{gathered}
m(y)=(2 T)^{-1} \int_{0}^{2 T}[F(x, y)-\mu] d x, \\
n(x)=(2 T)^{-1} \int_{0}^{2 T}[F(x, y)-\mu] d y, \\
\mu=(2 T)^{-2} \int_{0}^{2 T} \int_{0}^{2 T} F(x, y) d x d y,
\end{gathered}
$$

and take

$$
F_{0}(x, y)=m(y)+n(x)+\mu .
$$

Thus $m$ and $n$ are $2 T$-periodic functions of mean value zero, that is,

$$
\int_{0}^{2 T} m(y) d y=0, \quad \int_{0}^{2 T} n(x) d x=0 .
$$

Moreover, the function $F_{1}(x, y)$, or $F_{1}: \mathbb{R}^{2} \rightarrow \mathbb{R}$, defined by

$$
F_{1}(x, y)=F(x, y)-m(y)-n(x)-\mu=F(x, y)-F_{0}(x, y),
$$

is doubly $2 T$-periodic and has all its mean values zero, that is

$$
\begin{aligned}
\int_{0}^{2 T} F_{1}(x, y) d x=0 \text { for all } y, \quad \int_{0}^{2 T} F_{1}(x, y) d y=0 \text { for all } x, \\
\\
\int_{0}^{2 T} \int_{0}^{2 T} F_{1}(x, y) d x d y=0 .
\end{aligned}
$$

Finally, the function $U_{1}(x, y)$, or $U_{1}: \mathbb{R}^{2} \rightarrow \mathbb{R}$, defined by

$$
U_{1}(x, y)=\int_{0}^{x} \int_{0}^{y} F_{1}(\alpha, \beta) d \alpha d \beta,
$$

is again doubly $2 T$-periodic, with $U_{1}=0$ on the boundary of the fundamental square $S=\{(x, y) \mid 0 \leqq x \leqq 2 T, 0 \leqq y \leqq 2 T\}$. Note that $U_{1}=0$ also on all 
straight lines $[x=2 k T, y \in \mathbb{R}]$ and $[x \in \mathbb{R}, y=2 h T], h, k=0, \pm 1, \pm 2, \ldots$ Moreover,

$$
U_{1 x y}=F_{1}(x, y), \quad(x, y) \in \mathbb{R}^{2} .
$$

Any other doubly $2 T$-periodic continuous solution of equation (6) is of the form $U(x, y)=U_{1}(x, y)+\phi(x)+\psi(y)$, where $\phi, \psi$ are arbitrary $2 T$-periodic continuous functions, $\phi, \psi: \mathbb{R} \rightarrow \mathbb{R}$.

Now, let $F(x, y, U)$, or $F: \mathbb{R}^{3} \rightarrow \mathbb{R}$, be a given function which is doubly $2 T$-periodic in $x$ and $y$ and continuous in $\mathbb{R}^{3}$, and let us consider the problem of doubly $2 T$-periodic solutions $U(x, y)$ of the equation

$$
U_{x y}=F(x, y, U(x, y)) .
$$

As in CesARI [1] we shall express this problem in terms of functional analysis.

Let $X$ denote the Banach space of all doubly $2 T$-periodic functions $U(x, y)$ which are continuous in $\mathbb{R}^{2}$, and let $X$ be equipped with the uniform norm $\|U\|_{\infty}=\sup _{R^{2}}|U(x, y)|$. Let $X_{0}$ denote the subspace of all elements $U_{0}$ of $X$ of the form $U_{0}(x, y)=u_{0}(x)+v_{0}(y)$, where $u_{0}, v_{0}$ are $2 T$-periodic continuous functions in $\mathbb{R}$. Obviously, all constant functions are in $X_{0}$. Let $X_{1}$ denote the subspace of all elements $U_{1}$ of $X$ which are zero on the boundary $\partial S$ of the fundamental square $S$ (hence $U_{1}=0$ also on all the straight lines in $\mathbb{R}^{2}$ mentioned above).

If $P: X \rightarrow X$ is the projection operator defined by

$$
P U(x, y)=U(x, 0)+U(0, y)-U(0,0) \quad \text { for all }(x, y) \in \mathbb{R}^{2},
$$

then $U_{0}=P U$ is an element of $X_{0}$ and, furthermore, $P X=X_{0}$ and $(I-P) X$ $=X_{1}$, where $I$ is the identity map on $X$.

Let $Y=X$, with the same norm, and let $Y_{0}$ denote the subspace of all elements $F_{0}$ of $Y$ of the form $F_{0}(x, y)=m(y)+n(x)$, where $m, n$ are $2 T$-periodic continuous functions in $\mathbb{R}$ with mean value zero. Again, all constant functions are included in $Y_{0}$. Let $Y_{1}$ denote the subspace of $Y$ consisting of all functions $F_{1}(x, y)$ whose mean values are all zero (i.e., satisfy (3) and (4)).

Let $Q: Y \rightarrow Y$ denote the projection operator defined by

$$
Q F(x, y)=m(y)+n(x)+\mu \text { for all }(x, y) \in \mathbb{R}^{2},
$$

where $m, n, \mu$ are given by (1). Then $Q Y=Y_{0}, Y_{0}=X_{0}$, and $(I-Q) Y=Y_{1}$, where $I$ is again the identity map on $Y$.

Finally, let $H: Y_{1} \rightarrow X_{1}$ denote the linear operator defined by (5) for every element $F_{1} \in Y_{1}$, or $H F_{1}=U_{1}$.

We note that for any four points $(x, y),(\xi, \eta),(x, \eta),(\xi, y) \in \mathbb{R}^{2}$ we have

$$
U_{1}(x, y)+U_{1}(\xi, \eta)-U_{1}(x, \eta)-U_{1}(\xi, y)=\int_{\xi}^{x} \int_{\eta}^{y} F_{1}(\alpha, \beta) d \alpha d \beta,
$$

and if $\left\|F_{1}\right\|_{\infty} \leqq M$ also

$$
\left|U_{1}(x, y)+U_{1}(\xi, \eta)-U_{1}(x, \eta)-U_{1}(\xi, y)\right| \leqq M|x-\xi||y-\eta| .
$$


Thus, for $\eta=0,|y| \leqq T \cdot$ we have

$$
\left|U_{1}(x, y)-U_{1}(\xi, y)\right| \leqq M T|x-\xi|,
$$

and the same holds for $\eta=2 T, T \leqq|y| \leqq 2 T$.

By exchanging the roles of $x, \xi$ with $y, \eta$ we also get

$$
\begin{aligned}
& \left|U_{1}(x, y)-U_{1}(\xi, y)\right| \leqq M T|x-\xi|, \\
& \left|U_{1}(x, y)-U_{1}(x, \eta)\right| \leqq M T|y-\eta|,
\end{aligned}
$$

for all $(x, y),(\xi, y)$ and $(x, y),(x, \eta)$ in $\mathbb{R}^{2}$. Hence $U_{1}=H(I-Q) F$ is Lipschitz, continuous in $\mathbb{R}^{2}$ with Lipschitz constant $\sqrt{2} M T$.

It was proved by CESARI and KANNAN [4] that

$$
\begin{gathered}
\|P\|_{\infty}=3, \quad\|I-P\|_{\infty}=4, \quad\|Q\|_{\infty}=3, \\
\|I-Q\|_{\infty}=4, \quad\|H(I-Q)\|_{\infty}=T^{2},
\end{gathered}
$$

the last norm being the value of $T^{2} / 4$ in [4], with $2 T$ replacing $T$.

From relations (1), (2) we find that for $F \in Y$ the element $F_{1} \in Y_{1}$ has the integral representation

$$
\begin{aligned}
F_{1}(x, y) & =(I-Q) F(x, y) \\
& =(2 T)^{-2} \int_{0}^{2 T} \int_{0}^{2 T}[F(x, y)-F(\xi, y)-F(x, \eta)+F(\xi, \eta)] d \xi d \eta .
\end{aligned}
$$

Thus, if $F$ is Lipschitz continuous, say with

$$
|F(x+p, y)-F(x, y)| \leqq L|p|, \quad|F(x, y+q)-F(x, y)| \leqq L|q|,
$$

then

(10) $\left|F_{1}(x+p, y)-F_{1}(x, y)\right| \leqq 2 L|p|, \quad\left|F_{1}(x, y+q)-F_{1}(x, y)\right| \leqq 2 L|q|$.

\section{An Alternative Approach to Doubly T-Periodic Solutions$$
\text { of } u_{t t}-u_{\tau \tau}=f
$$

First let us consider the original problem concerning doubly $T$-periodic solutions $u(t, \tau)$ of the differential equation

$$
\Xi u \equiv u_{t t}-u_{\tau \tau}=\phi(t, \tau)+g(u),
$$

where $\phi$ is a given doubly $T$-periodic function. We shall assume $\phi$ continuous in $\mathbb{R}^{2}$ and $g$ continuous in $\mathbb{R}$, and we shall seek continuous solutions $u$.

Let $\Sigma$ denote the fundamental square, $\Sigma=[0 \leqq t, \tau \leqq T]$. Let $D$ denote the set of all doubly $T$-periodic functions $w(t, \tau)$ of class $C^{\infty}$ in $\mathbb{R}^{2}$. Let $\mathscr{X}$ denote the space of all continuous doubly $T$-periodic functions $u(t, \tau)$, with the uniform topology. Then $\mathscr{X}$ is a Banach space with the norm $\|u\|_{\infty}=\sup _{\mathbb{R}^{2}}|u(t, \tau)|$. 
Let $f \in \mathscr{X}$. An element $u \in \mathscr{X}$ is said to be a weak solution of $\Xi u=f$, or $u_{t t}-u_{\tau \tau}=f$, provided that

$$
\iint_{\Sigma}\left[\left(w_{t t}-w_{\tau \tau}\right) u+w f\right] d t d \tau=0 \text { for all } w \in D .
$$

The weak kernel of $\Xi$, or $\operatorname{ker} \Xi$, is the subspace $X_{0}$ of $\mathscr{X}$ made up of all functions $u \in \mathscr{X}$ of the form $u(t, \tau)=\phi(t+\tau)+\psi(t-\tau)$, where $\phi, \psi$ are $T$-periodic continuous functions in $\mathbb{R}$.

Let

$$
u(t, \tau)=\sum_{k, l=-\infty}^{+\infty} c_{k l} e^{(2 \pi i / T)(k t+l \tau)}
$$

be the complex form of the Fourier series of any element $u \in \mathscr{X}$. Then we define the projection operator $\mathscr{Q}: \mathscr{X} \rightarrow \mathscr{X}_{0}$ by

$$
\begin{aligned}
u_{0}(t, \tau)=\mathscr{Q} u(t, \tau) & =\sum_{|k|=|l|} c_{k l} e^{(2 \pi i / T)(k t+l \tau)} \\
& =c_{00}+\sum_{k \neq 0} c_{k k} e^{(2 \pi i / T) k(t+\tau)}+\sum_{l \neq 0} c_{l,-l} e^{(2 \pi i / T) l(t-\tau)}
\end{aligned}
$$

Thus $2 \mathscr{X}=\mathscr{X}_{0}=\operatorname{ker} \Xi$.

Let $\mathscr{X}_{1}=(I-\mathscr{2}) \mathscr{X}$, where $I$ is the identity map on $\mathscr{X}$. In other words, we consider elements $u_{1}$ of $\mathscr{X}$ represented by

$$
u_{1}(t, \tau)=(I-2) u(t, \tau)=\sum_{|k| \neq|l|} c_{k l} e^{(2 \pi i / T)(k t+l \tau)}
$$

for $u \in \mathscr{X}$ given by (1). We shall show that the operators $\mathscr{Q}$ and $I-\mathscr{Q}$ map functions of $\mathscr{X}$ into continuous functions; thus $\mathscr{Q}, I-\mathscr{Q}: \mathscr{X} \rightarrow \mathscr{X}, \mathscr{X}_{0}=\mathscr{Q} \mathscr{X}$, $\mathscr{X}_{1}=(I-\mathscr{Q}) \mathscr{X}$ and $\mathscr{X}_{0}=$ ker $\Xi$.

We have already considered the spaces $X=Y$ of continuous doubly $2 T$-periodic functions $U(x, y)$, or $U: \mathbb{R}^{2} \rightarrow \mathbb{R}$, and their decompositions $X=X_{0}+X_{1}$, $Y=Y_{0}+Y_{1}$. We have also considered the projection operators $P: X \rightarrow X$, $Q: Y \rightarrow Y$, with $P X=X_{0},(I-P) X=X_{1}, Q Y=Y_{0},(I-Q) Y=Y_{1}$.

Let $S=[0 \leqq x, y \leqq 2 T]$ be the fundamental square in the $x y$-space $\mathbb{R}^{2}$. For any $F \in Y$ the function $F_{0}(x, y)=Q F(x, y)=m(y)+n(x)+\mu$ is the system of the mean values of $F$, and $F_{1}=(I-Q) F$ is a function whose mean values are all zero. If

$$
F(x, y)=\sum_{k, l=-\infty}^{+\infty} c_{k l} e^{(n i / T)(k x+l y)} \text { for all }(x, y) \in \mathbb{R}^{2}
$$

is the Fourier series of $F$, which is certainly convergent to $F$ in $L_{2}(S)$, then

$$
\begin{aligned}
F_{0}(x, y) & =Q F(x, y)=m(y)+n(x)+\mu \\
& =(2 T)^{-2} \int_{0}^{2 T} \int_{0}^{2 T}[F(\xi, y)+F(x, \eta)-F(\xi, \eta)] d \xi d \eta \\
& =c_{00}+\sum_{k \neq 0} c_{k 0} e^{(\pi i / T) k x}+\sum_{l \neq 0} c_{0 l} e^{(\pi i / T) l y}
\end{aligned}
$$


Also

$$
F_{1}(x, y)=(I-Q) F(x, y)=\sum_{k \neq 0, l \neq 0} c_{k l} e^{(\pi i / T)(k x+l y)} .
$$

Note that the equation $u_{t t}-u_{v i}=\phi(t, \tau)+g(u), \phi, u \in \mathscr{X}$, is transformed by the operation $A$ into the equation

$$
U_{x y}=\Phi(x, y)+G(U), \quad \Phi, U \in X^{\prime},
$$

where $U(x, y)=u((x+y) / 2, \quad(x-y) / 2), \quad \Phi(x, y)=\frac{1}{4} \phi((x+y) / 2, \quad(x-y) / 2)$, $G(U)=\frac{1}{4} g(U)$, and where we have denoted by $X^{\prime}$ the image of $X$ in $X$ under the operation $A$, that is $X^{\prime}=A \mathscr{X}$.

We restate here that $X=Y$, that $X_{0}=Y_{0}$ is the space of all sums $V_{1}(x)$ $+V_{2}(y)$ of continuous $2 T$-periodic functions of $x$ only and of $y$ only, and that $P: X \rightarrow X_{0}, Q: Y \rightarrow Y_{0}=X_{0}$, though the operators $P$ and $Q$ are different. We shall identify the spaces $X$ and $Y$, so that now $Q$ can be thought of as mapping $X$ into $X_{0}$; of course $I-P$ maps $X$ into $X_{1}$ as usual, and $I-Q$ maps $X$ into the space $Y_{1}$, thought of as a subspace of $X$ different from $X_{1}$.

Also we indicate by $A$ the operation $u \rightarrow U$ defined by

$$
U(x, y)=u((x+y) / 2,(x-y) / 2),
$$

and by $A^{-1}$ the inverse operator $U \rightarrow u$, or

$$
u(t, \tau)=U(t+\tau, t-\tau)
$$

We denote by $X^{\prime}$ the subset of $X$ defined by $X^{\prime}=A \mathscr{X}$. Analogously, let $X_{0}^{\prime}, X_{1}^{\prime}$ denote the subsets of $X$ defined by $X_{0}^{\prime}=A X_{0}$ and $X_{1}^{\prime}=\Lambda X_{1}$. In other words, $X^{\prime}$ is the subspace of $X$ of all functions $U(x, y)$ in $X$ of the form $U(x, y)$ $=u((x+y) / 2,(x-y) / 2)$ with $u \in \mathscr{X}$, and analogously for $X_{0}^{\prime}$ and $X_{1}^{\prime}$. Clearly $X^{\prime}=X_{0}^{\prime}+X_{1}^{\prime}$. We shall denote by $j: X^{\prime} \rightarrow X$ the injection map, keeping in mind that $X=Y$. The space $X^{\prime}$, however, is smaller than $X$. For instance, $U(x, y)=\sin (\pi / T) x$ belongs to $X$ but not to $X^{\prime}$, since $u(t, \tau)=\sin (\pi / T)(t+\tau)$ is doubly $2 T$-periodic, but not doubly $T$-periodic. Finally, $X^{\prime}, X_{0}^{\prime}, X_{1}^{\prime}$ are closed subspaces of $X$ in the uniform topology. We now obtain some important relations.

(i) $X_{0}^{\prime} \subset X_{0}=Y_{0}, X_{1}^{\prime} \subset Y_{1}$.

Indeed, for $u \in \mathscr{X}$ we have

$$
u(t, \tau)=\sum_{k, l=-\infty}^{+\infty} c_{k l} e^{(2 \pi i / T)(k t+l \tau)}
$$

for $u \in \mathscr{X}_{0}$ we have

$$
\begin{aligned}
u(t, \tau) & =\sum_{|k|=|l|} c_{k l} e^{(2 \pi i / T)(k t+l \tau)} \\
& =c_{00}+\sum_{k \neq 0} c_{k k} e^{(2 \pi i / T) k(t+\tau)}+\sum_{l \neq 0} c_{l,-l} e^{(2 \pi i / T) l(t-\tau)}
\end{aligned}
$$


and for $u \in \mathscr{X}_{1}$ we have

$$
u(t, \tau)=\sum_{|k| \neq|l|} c_{k l} e^{(2 \pi i / T)(k t+l \tau)}
$$

Similarly; for $U \in \Lambda \mathscr{X}=X^{\prime}$ we have

$$
\begin{aligned}
U(x, y) & =\sum_{k, l=-\infty}^{+\infty} c_{k l} e^{(\pi i / T)[k(x+y)+l(x-y)]} \\
& =\sum_{k, l=-\infty}^{+\infty} c_{k l} e^{(\pi i / T)[(k+l) x+(k-l) y]}
\end{aligned}
$$

for $U^{*} \in A \mathscr{X}_{0}=X_{0}^{\prime}$ we have

$$
\begin{aligned}
U^{*}(x, y) & =\sum_{|k|=|l|} c_{k l} e^{(\pi i / T)[(k+l) x+(k-l) y]} \\
& =c_{00}+\sum_{k \neq 0} c_{k k} e^{(2 \pi i / T) k x}+\sum_{l \neq 0} c_{l,-l} e^{(2 \pi i / T) l y}
\end{aligned}
$$

and for $U_{1} \in \Lambda \mathscr{X}_{1}=X_{1}^{\prime}$ we have

$$
U_{1}(x, y)=\sum_{|k| \neq|l|} c_{k l} e^{(\pi i / T)[(k+l) x+(k-l) y]},
$$

where $k+l \neq 0, k-l \neq 0$. For $k^{\prime}=k+l, l^{\prime}=k-l$, we have $k^{\prime} \neq 0$, $l^{\prime} \neq 0$, and $k^{\prime}, l^{\prime}$ are either both even or both odd. If $\Sigma^{\prime}$ denotes a sum ranging over all such couples $\left(k^{\prime}, l^{\prime}\right)$, and $c_{k^{\prime}}^{\prime} l^{\prime}=c_{2^{-1}\left(k^{\prime}+l^{\prime}\right), 2^{-1}\left(k^{\prime}-l^{\prime}\right)}$, then

$$
U_{1}(x, y)=\Sigma^{\prime} c_{k^{\prime} l^{\prime}} e^{(\pi i / T)\left(k^{\prime} x+l^{\prime} y\right)}
$$

Comparing (6), (7), (8) with (3), (4), (5), we see that

$$
X^{\prime}=A \mathscr{X} \subset X, \quad X_{0}^{\prime}=A \mathscr{X}_{0} \subset X_{0}, \quad X_{1}^{\prime}=\Lambda \mathscr{X}_{1} \subset Y_{1},
$$

and the elements $U^{*} \in X_{0}^{\prime}=\Lambda \mathscr{X}_{0}$ are sums of $T$-periodic functions of $x$ only and of $y$ only.

(ii) $Q: X^{\prime} \rightarrow X_{0}^{\prime} ; \quad I-Q: X^{\prime} \rightarrow X_{1}^{\prime}$.

Indeed, any element $U \in X^{\prime}$ has a Fourier series (6), and the corresponding function $Q U$ given by (4) belongs to $X_{0}^{\prime}$; finally the corresponding function $(I-Q) U$ given by (5) belongs to $X_{1}^{\prime}$.

(iii) $2 u=\Lambda^{-1} Q \Lambda u$ for any $u \in \mathscr{X}$.

This can be best seen by using Fourier series. Thus for

$$
u(t, \tau)=\sum_{k, l=-\infty}^{+\infty} c_{k l} e^{(2 \pi i / T)(k t+l \tau)}
$$


we have

$$
\begin{gathered}
\Lambda u(x, y)=U(x, y)=\sum_{k, l=-\infty}^{+\infty} c_{k l} e^{(\pi i / T)[k(x+y)+l(x-y)]} \\
=\sum_{k, l=-\infty}^{+\infty} c_{k l} e^{(\pi i / T)[(k+l) x+(k-l) y]} \\
Q U(x, y)=c_{00}+\sum_{k \neq 0} c_{k k} e^{(2 \pi i / T) k x}+\sum_{l \neq 0} c_{l,-l} e^{(2 \pi i / T) l y} \\
A^{-1} Q U(t, \tau)=c_{00}+\sum_{k \neq 0} c_{k k} e^{(2 \pi i / T) k(t+\tau)}+\sum_{l \neq 0} c_{l,-l} e^{(2 \pi i / T) l(t-\tau)}
\end{gathered}
$$

Comparing this development with (2), we conclude that $\Lambda^{-1} Q \Lambda u=\mathscr{Q} u$ for any $u \in \mathscr{X}$. We also note that

$$
\begin{gathered}
(I-Q) U(x, y)=\sum_{|k| \neq|l|} c_{k l} e^{(\pi i / T)[(k+l) x+(k-t) y]}, \\
A^{-1}(I-Q) U(t, \tau)=\sum_{|k| \neq|l|} c_{k l} e^{(2 \pi i / T)(k t+l))} .
\end{gathered}
$$

(iv) $\mathscr{Q}: \mathscr{X} \rightarrow \mathscr{X}$ and $I-\mathscr{Q}: \mathscr{X} \rightarrow \mathscr{X}$ are continuous linear operations in $\mathscr{X}$ in the uniform topology.

Obviously $A$ and $\Lambda^{-1}$ are continuous linear operations from $\mathscr{X}$ to $X$ and from $X$ to $\mathscr{X}$ respectively. Thus, for $U \in X$ we have from (4),

$$
U_{0}(x, y)=Q U(x, y)=(2 T)^{-2} \int_{0}^{2 T} \int_{0}^{2 T}[U(\xi, y)+U(x, \eta)-U(\xi, \eta)] d \xi d \eta,
$$

and so $Q: X \rightarrow X_{0}$ is a continuous linear map.

(v) $(I-Q) H: X_{1}^{\prime} \rightarrow X_{1}^{\prime}$.

Let $E$ denote the operator defined by $E U=U_{x y}$, and note that $X_{0}$, the set of all sums of continuous $2 T$-periodic functions of $x$ only and of $y$ only, is the kernel of $E$ in $X$. Now, for any $U \in X_{1}^{\prime}$ we can write

$$
\begin{aligned}
U(x, y) & =\sum_{|k| \neq|l|} c_{k l} e^{(x i / T)[k(x+y)+l(x-y)]} \\
& =\sum_{|k| \neq|l|} c_{k l} e^{(\pi i / T)[(k+l) x+(k-l) y]},
\end{aligned}
$$

and consequently any solution $V$ of the equation $V_{x y}=U, V \in X$, must be of the form

(9) $V(x, y)=-\sum_{|k| \neq|l|}\left(T^{2} / \pi^{2}\right)[(k+l)(k-l)]^{-1} c_{k l} e^{(\pi i / T)[(k+l) x+(k-l) y]}+Z(x, y)$,

where $Z$ is any element of ker $E$. In other words

$$
V(x, y)=-\sum_{|k| \neq|l|}\left(T^{2} / \pi^{2}\right)\left(k^{2}-l^{2}\right)^{-1} e^{(\pi i / T)[(k+l) x+(k-l) y]}+V_{1}(x)+V_{2}(y),
$$


where $V_{1}(x), V_{2}(x)$ are arbitrary continuous $2 T$-periodic functions of $x$ only and of $y$ only. It follows that

$$
\begin{gathered}
Q V(x, y)=V_{1}(x)+V_{2}(y), \\
(I-Q) V(x, y)=-\sum_{|k| \neq|l|^{\prime}}\left(T^{2} / \pi^{2}\right)\left(k^{2}-l^{2}\right)^{-1} e^{(\pi i / T)[(k+l) x+(k-l) y]}, \\
A^{-1}(I-Q) V(t, \tau)=-\sum_{|k| \neq|l|}\left(T^{2} / \pi^{2}\right)\left(k^{2}-l^{2}\right)^{-1} e^{(2 \pi i \mid T)(k t+l \tau)},
\end{gathered}
$$

that is, $(I-Q) V=(I-Q) H U \in X_{1}^{\prime}$ for any $U \in X_{1}^{\prime}$.

(vi) $Q H(I-Q): X^{\prime} \rightarrow X_{0}=Y_{0}$.

For any $U \in X^{\prime}$, we have $(I-Q) U \in X_{1}^{\prime}$ by (ii). Hence by (v), $V=H(I-Q) U$ is a continuous doubly $2 T$-periodic solution of $V_{x y}=(I-Q) U$. Thus $V$ is of the form (9) and $Q V(x, y)=Z(x, y)=V_{1}(x)+V_{2}(y)$, that is, $Q H(I-Q) U \in X_{0}$.

Let $\mathrm{k}$ be a fixed real constant, which will ultimately depend on the particular problem under consideration. Also let $\mathscr{S}: y_{0} \rightarrow x_{0}$ be the identity map, and let $T^{*}$ denote the transformation defined by

$$
\begin{gathered}
\bar{U}_{1}=(I-Q) H(I-Q)\left[\Phi+G\left(U^{*}+U_{1}\right)\right]=K_{1} U \\
\bar{U}^{*}=U^{*}-k \mathscr{S} Q\left[\Phi+G\left(U^{*}+\bar{U}_{1}\right)\right]=K_{0} U,
\end{gathered}
$$

for all $U^{*} \in X_{0}^{\prime}, \quad U_{1} \in X_{1}^{\prime}$; clearly $U=U^{*}+U_{1} \in X^{\prime}$.

We shall denote by $N$ the nonlinear operator defined by $N U=\Phi+G(U)$.

(vii) For $U^{*} \in X_{0}^{\prime}$ and $U_{1} \in X_{1}^{\prime}$ we also have $\bar{U}^{*} \in X_{0}^{\prime}, \bar{U}_{1} \in X_{1}^{\prime}$.

Here $\Phi=\Lambda \phi$, where $\phi \in \mathscr{X}$; hence $\Phi \in X^{\prime}$. Now $U=U^{*}+U_{1} \in X^{\prime}$. Since $g$ is a continuous function, also $g(U) \in X^{\prime}$ and so $\Phi+G(U) \in X^{\prime}$. By (ii) and by (i) it follows that $(I-Q)[\Phi+G(U)] \in X_{1}^{\prime} \subset Y_{1}$, and by (v) we get $(I-Q) H(I-Q)[\Phi+G(U)] \in X_{1}^{\prime}$, that is, $\bar{U}_{1} \in X_{1}^{\prime}$. Therefore $U^{*}+$ $\bar{U}_{1} \in X^{\prime}$ and so $\Phi+G\left(U^{*}+\bar{U}_{1}\right) \in X^{\prime}$. By (ii) we have $Q\left[\Phi+G\left(U^{*}+\bar{U}_{1}\right)\right] \in X_{0}^{\prime}$. Since $U^{*} \in X_{0}^{\prime}$, it now follows that $\bar{U}^{*} \in X_{0}^{\prime}$.

(viii) If $U=U^{*}+U_{1}$, where $U^{*} \in X_{0}^{\prime}$ and $U_{1} \in X_{1}^{\prime}$, is a fixed point of $T^{*}$, then $U_{x y}=\Phi+G(U)$ and $u=\Lambda^{-1} U$ satisfies $u_{t t}-u_{\tau \tau}=\phi+g(u)$.

First, $Q[\Phi+G(U)]=0$. Now $(I-Q) H(I-Q)[\Phi+G(U)] \in X_{1}^{\prime}$ as noted above in the proof of (vii). Moreover, by (vi), $Q H(I-Q)[\Phi+G(U)] \in X_{0}$ is a sum of $2 T$-periodic continuous functions of $x$ only and of $y$ only. Also $U^{*}$ is a sum of continuous $2 T$-periodic functions of $x$ only and of $y$ only. Thus $U \in$ $X^{\prime}=\Lambda \mathscr{X}$ and

$$
\begin{aligned}
U_{x y} & =U_{x y}^{*}+U_{1 x y}=0+U_{1 x y} \\
& =[H(I-Q)(\Phi+G(U))]_{x y}-[Q H(I-Q)(\Phi+G(U))]_{x y} \\
& =(I-Q)[\Phi+G(U)]-0=\Phi+G(U) .
\end{aligned}
$$

Hence we have

$$
u_{t t}-u_{x \tau}=\phi+g(u), \quad \text { for } u=\Lambda^{-1} U .
$$




\section{A Suitable Mixed Uniform-Lipschitz Topology}

Let $X_{0}^{\prime *}$ be the subspace of all elements $U^{*} \in X_{0}^{\prime}$ which are Lipschitz continuous in $\mathbb{R}^{2}$. Since $U^{*}(x, y)=V_{1}(x)+V_{2}(y)$ is a sum of functions of $x$ only and of $y$ only, this is actually a restriction to functions $V_{1}(x), V_{2}(x)$ which are Lipschitz continuous in $\mathbb{R}$. For any Lipschitz continuous function $U(x, y)$, or $U: \mathbb{R}^{2} \rightarrow \mathbb{R}$, let

$$
\begin{aligned}
& \operatorname{Lip}_{x} U=\sup |p|^{-1}|U(x+p, y)-U(y, x)|, \\
& \operatorname{Lip}_{y} U=\sup |q|^{-1}|U(x, y+q)-U(x, y)| .
\end{aligned}
$$

Finally we denote by $\left\|\left|U^{*}\right|\right\|$ the new norm in $X_{0}^{\prime *}$ :

$$
\left\|U^{*}\right\|\|=\| U^{*}\left\|_{\infty}+A \max \left[\operatorname{Lip}_{x} U^{*}, \operatorname{Lip}_{y} U^{*}\right]=\right\| U^{*}\left\|_{\infty}+A\right\| U^{*} \|_{*},
$$

a mixed uniform-Lipschitz norm, where $A$ is a fixed constant, which will ultimately depend on the particular problem under consideration.

For given $R_{0}, r>0$, let us consider the sets

$$
\begin{gathered}
S_{0}^{*}=\left\{U^{*} \in X_{0}^{\prime *}:\|\| U^{*} \| \mid \leqq R_{0}\right\}, \quad S_{1}=\left\{U_{1} \in X_{1}^{\prime}:\left\|U_{1}\right\|_{\infty} \leqq r\right\}, \\
\Omega^{*}=S_{0}^{*} \times S_{1} .
\end{gathered}
$$

We shall restrict $T^{*}$ to the set $\Omega^{*}$ and shall prove below that, under suitable hypotheses, $T^{*}$ maps $\Omega^{*}$ into itself and that $T^{*}$ has a fixed point in $\Omega^{*}$, that is, $U=$ $U^{*}+U_{1} \in \Omega^{*}$ with $T^{*} U=U$.

We consider $\Omega^{*}$ as a subset of the Banach space $X$ with norm $\|\cdot\|_{\infty}$. We first show that $\Omega^{*}$ is closed in the uniform topology. Let $U_{k}=U_{k}^{*}+U_{k 1}, k=1,2, \ldots$ be in $\Omega *$ with $U_{k} \rightarrow U$, i.e., $\left\|U_{k}-U\right\|_{\infty} \rightarrow 0$ as $k \rightarrow \infty$. Then

$$
\left\|U_{k 1}\right\|_{\infty} \leqq r, \quad\left\|U_{k}^{*}\right\|_{\infty}+A\left\|U_{k}^{*}\right\|_{*} \leqq R_{0} .
$$

Thus the elements $U_{k}^{*}$ are uniformly bounded and uniformly Lipschitz continuous. Hence $U_{k}^{*} \rightarrow U^{*}$, where $U^{*}$ is Lipschitz continuous and

$$
\left\|U^{*}\right\|_{*} \leqq \lim \inf \left\|U_{k}^{*}\right\|_{*}, \quad\left\|U^{*}\right\|_{\infty} \leqq \lim \inf \left\|U_{k}^{*}\right\|_{\infty},
$$

implying that $\left\|U^{*}\right\|_{\infty}+A\left\|U^{*}\right\|_{*} \leqq R_{0}$. Analogously, $U_{k 1} \rightarrow U_{1}$ as $k \rightarrow \infty$ and $\left\|U_{1}\right\|_{\infty} \leqq \lim \inf \left\|U_{k 1}\right\|_{\infty} \leqq r$. Thus $\Omega^{*}$ is closed in $X$.

It is easily seen that $\Omega^{*}$ is convex.

We now show that $T^{*}: \Omega^{*} \rightarrow \Omega^{*}$ is compact in the topology of $X$. In fact, if $U_{k} \in \Omega^{*}, \bar{U}_{k}=T^{*} U_{k}, \bar{U}_{k} \in \Omega^{*}$ with $\bar{U}_{k}=\bar{U}_{k}^{*}+\bar{U}_{k 1}, k=1,2, \ldots$, then

$$
\bar{U}_{k 1}=(I-Q) H(I-Q) N\left(U_{k}\right)=(I-Q) H(I-Q) N\left(U_{k}^{*}+U_{k 1}\right) .
$$

Thus for $|\phi| \leqq c, \quad|g(u)| \leqq C$, we have $|\Phi+G(U)| \leqq \frac{1}{4}(c+C)$. Since $\|H(I-Q)\|_{\infty} \leqq T^{2}$ and $\|I-Q\|_{\infty}=4$, we obtain

$$
\left\|\bar{U}_{k 1}\right\|_{\infty} \leqq(c+C) T^{2}
$$

On the other hand, $H(I-Q) N\left(U_{k}\right)$ is Lipschitz continuous with constant $\frac{1}{4}(c+C) T$; hence $\bar{U}_{k 1}$ is Lipschitz continuous with constant $\frac{1}{2}(c+C) T$ by relations (8) and (10) of $\S 3$. Now by hypothesis $\bar{U}_{k}^{*}$ is Lipschitz continuous with constant $R / A$, and hence $\bar{U}_{k}$ is Lipschitz continuous with constant $(R / A)+$ 
$\frac{1}{2}(c+C) T$. Moreover $\left\|\bar{U}_{k}^{*}\right\|_{\infty} \leqq R_{0},\left\|\bar{U}_{k 1}\right\|_{\infty} \leqq(c+C) T^{2}$, so that

$$
\left\|\bar{U}_{k}\right\|_{\infty} \leqq R_{0}+(c+C) T^{2} \text {. }
$$

Thus, by Ascoli's theorem there is a subsequence $\left(\bar{U}_{k_{s}}\right)$ which is uniformly convergent to some $U \in X$.

It follows that $T^{*}$ is a compact map in the topology of $X$, provided we prove that $T^{*}$ maps $\Omega^{*}$ into itself. By Schauder's fixed point theorem, $T^{*}$ has at least one fixed point $U$ in $\Omega^{*}$, i.e., the problems (1) and (2) of $\S 1$ have at least one Lipschitz continuous periodic solution.

Theorem. Let $\phi: \mathbb{R}^{2} \rightarrow \mathbb{R}$ and $g: \mathbb{R} \rightarrow \mathbf{R}$ satisfy the following assumptions:

(1) $\phi(t+T, \tau)=\phi(t, \tau)=\phi(t, \tau+T)$ for all $(t, \tau) \in \mathbb{R}^{2}$;

(2) $|\phi(t, \tau)| \leqq c,|g(u)| \leqq C$, for all $(t, \tau) \in \mathbb{R}^{2},|u| \leqq R_{0}+r$;

(3) $g(u) \geqq B$ for $u \geqq b, g(u) \leqq-B$ for $u \leqq-b, g(0)=0$;

(4) $|\phi(t, \tau)-\phi(t+\bar{h}, \tau+\bar{h})-\phi(t+\bar{k}, \tau-\bar{k})+\phi(t+\bar{h}+\bar{k}, \tau+\bar{h}-\bar{k})|$ $\leqq c^{\prime}$ for all $t, \tau, \bar{h}, \bar{k} \in \mathbb{R}$

(5) $|\phi(t, \tau)-\phi(t+\bar{h}+\bar{k}, \tau+\bar{h}-\bar{k})| \leqq 2 \lambda\left(\overline{h^{2}}+\overline{k^{2}}\right)^{1 / 2}$ for all $t, \tau, \bar{h}, \bar{k} \in \mathbb{R}$;

(6) $|g(u)-g(v)| \leqq D|u-v|$ for all $|u|,|v| \leqq R_{0}+r$;

(7) $|g(u)-g(v)| \leqq d|u-v|$ for all $|u|,|v| \leqq R_{0}+r$ and either $u, v \geqq b$ or $u, v \leqq-b$;

where $b, c, c, B, C, d, D, r, R_{0}, \lambda$ are given positive constants satisfying

$$
B<C, \quad d<D .
$$

Assume also that there the constant $k$ in (11), Section 4, and the constant $A$ above satisfy the following conditions:

(8) $|u-v-k g(u)+k g(v)| \leqq \gamma|u-v|$ for all $|u|,|v| \leqq v$,

(9) $T^{2}(c+C) \leqq r$

(10) $R_{0}-R_{1}+r \leqq v$;

(11) $R_{0}-R_{1}-2\left(R_{1} / A\right) T-r \geqq b$;

(12) $c+c^{\prime}+\lambda A+2\left(R_{1} / A\right) d T+(c+C) d T^{2}+d R_{1}+\frac{1}{2} d(c+C) T A \leqq B$;

(13) $k\left[\frac{1}{4}\left(c+c^{\prime}+C+\lambda A\right)+\frac{1}{4}(c+C) d T^{2}+\frac{1}{\varepsilon} d(c+C) T A\right] \leqq 2\left(R_{0}-R_{1}\right.$ $\left.-\left(R_{1} / A\right) T\right)-\frac{1}{2} k\left(R_{1} / A\right) d T-\frac{1}{4} k d R_{1}$

(14) $k\left(c+c^{\prime}+\lambda A+D r\right)+2(c+C) T+(c+C) T^{2}+2(c+C) T A$ $+\frac{1}{2} \gamma(c+C) T A \leqq(1-\gamma-4 \gamma T / A) R_{0}$.

where $\gamma, v$ and $R_{1}$ are positive constants satisfying

$$
b<v<R_{0}+r, \quad R_{1}<R_{0} .
$$

Then the nonlinear problem

$$
u_{t t}-u_{z \tau}=\phi(t, \tau)+g(u)
$$

has at least one continuous doubly T-periodic weak solution.

Proof. Let us write $R$ instead of $R_{1}$ in relations (11), (12), (13), and note that such relations then hold for all $0 \leq R \leq R_{1}$. Let us denote the same relations, 
with $R$ replacing $R_{1}$, by the same numbers. Multiplying the new relation (12) by $k / 4$ we have

$$
\begin{aligned}
& \begin{aligned}
\frac{1}{4} k\left(c+c^{\prime}+\lambda A\right)+\frac{1}{2} k(R / A) d T+ & \frac{1}{4} k(c+C) d T^{2}+\frac{1}{4} k d R+\frac{1}{8} k d(c+C) T A \\
& \leqq \frac{1}{4} k B,
\end{aligned} \\
& \text { or, equivalently, }
\end{aligned}
$$

$$
\begin{aligned}
R_{0}-\frac{1}{4} k B+\frac{1}{2} k(R / A) d T & +\frac{1}{4} k d R+\frac{1}{4} k\left[c+c^{\prime}+\lambda A+(c+C) d T^{2}\right. \\
& \left.+\frac{1}{2} d(c+C) T A\right] \leqq R_{0}
\end{aligned}
$$

By adding and subtracting $R$ and rearranging the terms, we also get

$$
\begin{gathered}
{\left[R_{0}-R-\frac{1}{4} k B+\frac{1}{4} k\left(c+c^{\prime}\right)+\frac{1}{2} k(R / A) d T+\frac{1}{4} k(c+C) d T^{2}\right]} \\
+\left(1+\frac{1}{4} k d\right) R+\frac{1}{8} k d(c+C) T A+\frac{1}{4} k \lambda A \leqq R_{0},
\end{gathered}
$$

in other words,

and thus

$$
Z_{1}+\left(1+\frac{1}{4} k d\right) R+\frac{1}{4} k \lambda A+\frac{1}{8} k d(c+C) T A \leqq R_{0},
$$

$$
\begin{aligned}
& Z_{1}=R_{0}-R\left(1-\frac{1}{2} k d T / A\right)+\frac{1}{4} k\left(c+c^{\prime}-B\right)+\frac{1}{4} k(c+C) d T^{2} \leqq R_{0} . \\
& \text { Now, if } \\
& Z_{2}=R_{0}-R\left(1+2 T / A+\frac{1}{2} k d T / A\right)-\frac{1}{4} k\left(c+c^{\prime}+C\right)-\frac{1}{4} k(c+C) d T^{2}
\end{aligned}
$$

is negative, we let $Z_{2}^{-}=-Z_{2}$ and from (13) we derive

$$
\begin{aligned}
& -R_{0}+R+2(R / A) T+\frac{1}{4} k\left(c+c^{\prime}+C+\lambda A\right)+\frac{1}{2} k(R / A) d T \\
& +\frac{1}{4} k(c+C) d T^{2}+R\left(1+\frac{1}{4} k d\right)+\frac{1}{8} k d(c+C) T A \leqq R_{0},
\end{aligned}
$$

in other words

and

$$
Z_{2}^{-}+R\left(1+\frac{1}{4} k d\right)+\frac{1}{8} k d(c+C) T A+{ }_{4}^{1} k \lambda A \leqq R_{0}
$$

$Z_{2}=R_{0}-R\left(1+2 T / A+\frac{1}{2} k d T / A\right)-\frac{1}{4} k\left(c+c^{\prime}+C\right)-\frac{1}{4} k(c+C) d T^{2} \geqq-R_{0}$.

If $Z_{2}>0$, then $Z_{2}^{-}=0$ and the above relations are still true.

Finally, multiplying (14) by $\frac{1}{4}$ we derive

in other words

$$
\begin{gathered}
\frac{1}{4} k\left(D r+c+c^{\prime}+\lambda A\right)+\frac{1}{2}(c+C) T+\frac{1}{4}(c+C) T^{2} \\
+\frac{1}{2}\left(1+\frac{1}{4} \gamma\right)(c+C) T A \leqq\left(\frac{1}{4}-\frac{1}{4} \gamma-\gamma T / A\right) R_{0},
\end{gathered}
$$

$$
\begin{gathered}
\gamma\left(\frac{1}{4}+T / A\right) R_{0}+\frac{3}{4} R_{0}+\frac{1}{4} k\left(D r+c+c^{\prime}+\lambda A\right)+\frac{1}{2}(c+C) T\left[1+\frac{1}{2} T\right. \\
\left.+\left(1+\frac{1}{4} \gamma\right) A\right] \leqq R_{0} .
\end{gathered}
$$

Note that for $\phi \in \operatorname{ker} \Xi$ we have $c^{\prime}=0$, for otherwise certainly $c^{\prime} \leqq 4 c$.

As noted earlier, the proof is complete if we show that $T^{*}$ maps $\Omega^{*}$ into itself. Recall that $T^{*}$ is defined by $(10)$ and $(11)$ of $\S 4$, with $T^{*}:\left(U^{*}, U_{1}\right) \rightarrow\left(\bar{U}^{*}, \bar{U}_{1}\right)$.

By assumption (2) and by the fact that

and

$$
\Phi(x, y)=\frac{1}{4} \phi((x+y) / 2,(x-y) / 2)
$$

$$
G(U(x, y))=\frac{1}{4} g(u((x+y) / 2,(x-y)) / 2),
$$


we get $\|N(U)\|_{\infty}=\|\Phi+G(U)\|_{\infty} \leqq \frac{1}{4}(c+C)$. Also

$$
\|m\|_{\infty} \leqq \frac{1}{2}(c+C),\|n\|_{\infty} \leqq \frac{1}{2}(c+C), \quad|\mu| \leqq \frac{1}{4}(c+C)
$$

and from relation (1) of $\S 3$, as in [4],

$$
\|m+n+\mu\|_{\infty} \leqq \frac{3}{4}(c+C),\|(I-Q) N U\|_{\infty} \leqq c+C .
$$

Thus from relations (9) of $\$ 3$ we again have

$$
\left\|\vec{U}_{1}\right\|_{\infty}=\left\|K_{1} U\right\|_{\infty}=\|(I-Q) H(I-Q) N(U)\|_{\infty} \leqq T^{2}(c+C) \leqq r
$$

for all $U=U^{*}+U_{1} \in \Omega^{*}$. Therefore $K_{1}: \Omega^{*} \rightarrow S_{1}$.

Moreover, from (8) and (10) of $\S 3$ we derive

$$
\max \left[\operatorname{Lip}_{x} \bar{U}_{1}, \operatorname{Lip}_{y} \bar{U}_{1}\right] \leqq \frac{1}{2} T(c+C) .
$$
sion

Before we discuss the map $K_{0}$, defined by (11) of $\S 4$, we consider the expres-

$$
\bar{U}^{*}=U^{*}-k \mathscr{S Q N}\left(U^{*}+\bar{U}_{1}\right),
$$

where $U=U^{*}+U_{1} \in \Omega^{*}$ and $\bar{U}_{1}=K_{1} U$. Calling $U^{\prime}=U^{*}+\bar{U}_{1}$, we have

$$
\begin{aligned}
& \bar{U}^{*}(x, y)=U^{*}(x, y)-k \mathscr{S Q N U} U^{\prime}(x, y) \\
& =U^{*}(x, y)-k(2 T)^{-1} \int_{0}^{2 T}\left[\Phi(\xi, y)+G\left(U^{\prime}(\xi, y)\right)\right] d \xi \\
& -k(2 T)^{-1} \int_{0}^{2 T}\left[\Phi(x, \eta)+G\left(U^{\prime}(x, \eta)\right)\right] d \eta \\
& +k(2 T)^{-2} \int_{0}^{2 T} \int_{0}^{2 T}\left[\Phi(\xi, \eta)+G\left(U^{\prime}(\xi, \eta)\right)\right] d \xi d \eta
\end{aligned}
$$

or, equivalently,

$$
\begin{aligned}
\bar{U}^{*}(x, y)= & U^{*}(x, y)-k\left[\Phi(x, y)+G\left(U^{\prime}(x, y)\right)\right] \\
& +k(2 T)^{-2} \int_{0}^{2 T} \int_{0}^{2 T}[\Delta \Phi+\Delta G] d \xi d \eta,
\end{aligned}
$$

where for the sake of brevity we have put

$$
\begin{gathered}
\Delta \Phi=\Phi(x, y)-\Phi(\xi, y)-\Phi(x, \eta)+\Phi(\xi, \eta) \\
\Delta G=G\left(U^{\prime}(x, y)\right)-G\left(U^{\prime}(\xi, y)\right)-G\left(U^{\prime}(x, \eta)\right)+G\left(U^{\prime}(\xi, \eta)\right) .
\end{gathered}
$$

If we write $\xi=x+2 \bar{h}, \eta=y+2 \bar{k}$, then from assumptions (4) and (5) follows$$
|\Delta \Phi|=|\Phi(x, y)-\Phi(x+2 h, y)-\Phi(x, y+2 \bar{k})+\Phi(x+2 \bar{h}, y+2 \bar{k})|
$$$$
=\mid \frac{1}{4}\left[\phi\left(\frac{1}{2}(x+y), \frac{1}{2}(x-y)\right)-\phi\left(\frac{1}{2}(x+y)+\bar{h}, \frac{1}{2}(x-y)+\bar{h}\right)\right.
$$$$
\left.-\phi\left(\frac{1}{2}(x+y)+\bar{k}, \frac{1}{2}(x-y)-\bar{k}\right)+\phi\left(\frac{1}{2}(x+y)+\bar{h}+k, \frac{1}{2}(x-y)+\bar{h}-\bar{k}\right)\right]
$$$$
=1 \frac{1}{4}[\phi(t, \tau)-\phi(t+\bar{h}, \tau+\bar{h})-\phi(t+\bar{k}, \tau-\bar{k})
$$$$
+\phi(t+\bar{h}+\bar{k}, \tau+\bar{h}-\bar{k})] \mid \leqq \frac{1}{4} c^{\prime} \text {; }
$$ 


$$
\begin{aligned}
|\Phi(x, y)-\Phi(x+2 \bar{h}, y+2 \bar{k})| \\
=\left|\frac{1}{4}\left[\phi\left(\frac{1}{2}(x+y), \frac{1}{2}(x-y)\right)-\phi\left(\frac{1}{2}(x+y)+h+\bar{k}, \frac{1}{2}(x-y)+\bar{h}-\bar{k}\right)\right]\right| \\
\quad=\left|\frac{1}{4}[\phi(t, \tau)-\phi(t+\bar{h}+\bar{k}, \tau+\bar{h}-\bar{k})]\right| \leqq \frac{1}{4} \cdot 2 \lambda\left(\overline{h^{2}}+\overline{k^{2}}\right)^{\frac{1}{2}} \\
\quad=\frac{1}{4} \lambda\left[(2 \bar{h})^{2}+(2 \bar{k})^{2}\right]^{\frac{1}{2}},
\end{aligned}
$$

that is,

$$
|\Phi(P)-\Phi(Q)| \leqq \frac{1}{4} \lambda|P-Q|
$$

Two cases arise in the study of the map $K_{0}$.

Case 1. $U=U^{*}+U_{1} \in \Omega^{*},\left\|U^{*}\right\|_{\infty}=R_{0}-R, \quad \max \left[\operatorname{Lip}_{x} U^{*}, \operatorname{Lip}_{y} U^{*}\right]$ $\leqq R / A$ for some $R, 0 \leqslant R \leqslant R_{1}<R_{0}$. Observe that

$$
\left\|U^{*}\right\|=\left\|U^{*}\right\|_{\infty}+A\left\|U^{*}\right\|_{*} \leqq R_{0}-R+A(R / A)=R_{0}
$$

and $\left\|U_{1}\right\|_{\infty} \leqq r,\left\|\bar{U}_{1}\right\|_{\infty} \leqq r$, as shown in (15).

Note that $U^{*} \in S_{0}^{*}$ is a function of the form $U^{*}(x, y)=U_{0}(x)+V_{0}(y)$ with $\left\|U^{*}\right\|_{* *}=\left\|U_{0}+V_{0}\right\|_{*} \leqq R / A$ by assumption. Thus

$$
\text { Osc }\left[U_{0}(x), 0 \leqq x \leqq 2 T\right] \leqq(R / A) T
$$

since $U_{0}(0)=U_{0}(T)=U_{0}(2 T)$. Analogously,

$$
\text { Osc }\left[V_{\mathrm{o}}(y), 0 \leqq y \leqq 2 T\right] \leqq(R / A) T .
$$

Hence $U^{*}$ has the same value at the points $(r T, s T), r, s=0, \pm 1, \pm 2, \ldots$, and moreover

$$
\operatorname{Osc}\left[U^{*}(x, y), 0 \leqq x, y \leqq 2 T\right] \leqq 2(R / A) T
$$

It follows that

$$
\left|U^{*}(x, y)\right| \geqq\left\|U^{*}\right\|_{\infty}-2(R / A) T \geqq R_{0}-R-2(R / A) T \text { for all }(x, y) \in \mathbb{R}^{2} .
$$

Then, by (15) and by assumption (11) we also derive

$$
\begin{gathered}
\left|U^{\prime}(x, y)\right|=\left|U^{*}(x, y)+\bar{U}_{1}(x, y)\right| \geqq R_{0}-R-2(R / A) T-r \geqq b \\
\text { for } U^{\prime}=U^{*}+\bar{U}_{1} .
\end{gathered}
$$

Therefore either $U^{\prime}(x, y) \geqq b$ for all $(x, y) \in \mathbf{R}^{2}$ or $U^{\prime}(x, y) \leqq-b$ for all $(x, y) \in \mathbb{R}^{2}$.

If $U^{\prime}(x, y) \geqq b$ for all $(x, y)$, then by using assumption (3) we obtain

$$
g\left(U^{\prime}(x, y)\right) \geqq B \text { for all }(x, y) .
$$

With this in mind, we now consider the expression (17), where the integration over $[0,2 T] \times[0,2 T]$ can be performed instead over $[x-T, x+T] \times[y-T$, $y+T]$, because of the double $2 T$-periodicity of the integrand.

The expressions $\Delta \Phi, \Delta G$ in the integrand can now be estimated. First by using assumption (4), we get $|\Delta \Phi| \leqq \frac{1}{4} c^{\prime}$, as stated above. Next, since any two corresponding points in $\Delta G$ are equivalent to points at a distance less or equal to 
$T$ because of the double $2 T$-periodicity, by using assumption (7), inequality (16), and the fact $\left\|U^{*}\right\|_{*} \leqq R / A$ we obtain

$$
\begin{aligned}
|\Delta G| & \leqq \frac{1}{4} d\left[R / A+\frac{1}{2}(c+C) T\right] T+\frac{1}{4} d\left[R / A+\frac{1}{2}(c+C) T\right] T \\
& =\frac{1}{2} d T(R / A)+\frac{1}{4}(c+C) d T^{2} .
\end{aligned}
$$

Thus from (17) and the above relations,

$$
\begin{aligned}
\bar{U}^{*}(x, y)= & U^{*}(x, y)-k \Phi(x, y)-k G\left(U^{\prime}(x, y)\right) \\
& +k(2 T)^{-2} \int_{x-T}^{x+T} \int_{y-T}^{y+T}[\Delta \Phi+\Delta G] d \xi d \eta,
\end{aligned}
$$

$\bar{U}^{*}(x, y) \leqq R_{0}-R+k \frac{1}{4} c-k \frac{1}{4} B+k \frac{1}{4} c^{\prime}+\frac{1}{2} k d T(R / A)+\frac{1}{4} k(c+C) d T^{2}=Z_{1}$

$$
=R_{0}-R\left(1-\frac{1}{2} k d T / A\right)+\frac{1}{4} k\left(c+c^{\prime}-B\right)+\frac{1}{4} k(c+C) d T^{2} \leqq R_{0},
$$

$\bar{U}^{*}(x, y) \geqq R_{0}-R-2(R / A) T-k \frac{1}{4} c-k \frac{1}{4} C$

$$
\begin{aligned}
& -k\left[\frac{1}{4} c^{\prime}+\frac{1}{2} d(R / A) T+\frac{1}{4}(c+C) d T^{2}\right]=Z_{2} \\
= & R_{0}-R\left(1+2 T / A+\frac{1}{2} k d T / A\right)-\frac{1}{4} k\left(c+c^{\prime}+C\right) \\
& -\frac{1}{4} k(c+C) d T^{2} \geqq-R_{0} .
\end{aligned}
$$

If $U^{\prime}(x, y) \leqq-b$ for all $(x, y)$, then by assumption (3) we have $g\left(U^{\prime}(x, y)\right) \leqq$ $-B$. By using the same argument, we also get $-R_{0} \leqq \bar{U}^{*}(x, y) \leqq R_{0}$ for all $(x, y)$.

To obtain the Lipschitz constant of $\bar{U}^{*}$, we note that

$$
\begin{aligned}
\vec{U}^{*}(x, y)= & U^{*}(x, y)-k[m(y)+n(x)+\mu] \\
= & -k[m(y)+\mu]+U^{*}(x, y)-k(2 T)^{-1} \int_{0}^{2 T} \Phi(x, \eta) d \eta \\
& -k(2 T)^{-1} \int_{0}^{2 T} G\left(U^{\prime}(x, \eta)\right) d \eta ;
\end{aligned}
$$

thus

$$
\begin{aligned}
\bar{U}^{*}(x+p, y)-\bar{U}^{*}(x, y)= & U^{*}(x+p, y)-U^{*}(x, y) \\
& -k(2 T)^{-1} \int_{0}^{2 T}[\Phi(x+p, \eta)-\Phi(x, \eta)] d \eta \\
& -k(2 T)^{-1} \int_{0}^{2 T}\left[G\left(U^{\prime}(x+p, \eta)\right)-G\left(U^{\prime}(x, \eta)\right)\right] d \eta .
\end{aligned}
$$

Hence, by assumptions (5) and (7) and since $\left\|U^{*}\right\|_{*} \leqq R / A$, we have

$$
\begin{aligned}
\left|\bar{U}^{*}(x+p, y)-\bar{U}^{*}(x, y)\right| \leqq & (R / A)|p|+k_{4}^{1} \lambda|p| \\
& +k(2 T)^{-1} \int_{0}^{2 T} \frac{1}{4} d\left|U^{\prime}(x+p, \eta)-U^{\prime}(x, \eta)\right| d \eta \\
\leqq & {\left[(R / A)+\frac{1}{4} k \lambda+\frac{1}{4} k d\left(R / A+\frac{1}{2}(c+C) T\right)\right]|p| . }
\end{aligned}
$$


Thus

$$
\operatorname{Lip}_{x} \bar{U}^{*} \leqq(R / A)\left(1+\frac{1}{4} k d\right)+\frac{1}{4} k \lambda+\frac{1}{8} k d(c+C) T,
$$

and the same inequality holds for $\operatorname{Lip}_{y} \bar{U}^{*}$. Therefore

$$
\begin{aligned}
\left\|\bar{U}^{*}\right\| & =\left\|\bar{U}^{*}\right\|_{\infty}+A\left\|\bar{U}^{*}\right\|_{*} \\
& \leqq \max \left[Z_{1}, Z_{2}^{-}\right]+R\left(1+\frac{1}{4} k d\right)+\frac{1}{4} k \lambda A+\frac{1}{8} k d(c+C) T A .
\end{aligned}
$$

The requirement \|\| $\bar{U}^{*}\|\| \leqq R_{0}$, i.e., $\bar{U}^{*} \in S_{0}^{*}$, is satisfied provided assumptions (12) and (13) are verified, as stated above.

Case 2. $U=U^{*}+U_{1} \in \Omega^{*},\left\|U^{*}\right\|_{\infty}=R_{0}-R,\left\|U^{*}\right\|_{*} \leqq R / A$ for some $R$, $0<R_{1} \leqslant R \leqslant R_{0}$. Again

$$
\left\|U^{*}\right\| \mid \leqq R_{0},\left\|U_{1}\right\|_{\infty} \leqq r,\left\|\bar{U}_{1}\right\|_{\infty} \leqq r,\left\|\bar{U}_{1}\right\|_{*} \leqq \frac{1}{2}(c+C) T .
$$

Again, let us write $R$ instead of $R_{1}$ in relation (10), and note that the new relation holds for all $R$ with $R_{1} \leqslant R \leqslant R_{0}$. Let us denote the new relation by the same number. By the new relation (10) we have

(18) $\left\|U^{*}\right\|_{\infty}=R_{0}-R<\nu, \quad\left\|U^{\prime}\right\|_{\infty}=\left\|U^{*}+\bar{U}_{1}\right\|_{\infty} \leqq R_{0}-R+r \leqq \nu$,

and by (8) for $v=0$ we also get

$$
\left\|U^{*}-k g\left(U^{*}\right)\right\|_{\infty} \leqq \gamma\left\|U^{*}\right\|_{\infty} \leqq \gamma\left(R_{0}-R\right) .
$$

Since $v<R_{0}+r$, we have by assumptions (6) and (8)

$$
\begin{aligned}
& \left\|g\left(U^{*}\right)-g\left(U^{\prime}\right)\right\|_{\infty} \leqq D\left\|\bar{U}_{1}\right\|_{\infty} \leqq D r, \\
\left\|U^{*}-k g\left(U^{\prime}\right)\right\|_{\infty} & \leqq \frac{1}{4}\left\|U^{*}-k g\left(U^{*}\right)\right\|_{\infty}+\left(\frac{3}{4}\right)\left\|U^{*}\right\|_{\infty}+k\left\|G\left(U^{*}\right)-G\left(U^{\prime}\right)\right\|_{\infty} \\
& \leqq \frac{1}{4} \gamma\left(R_{0}-R\right)+\left(\frac{3}{4}\right)\left(R_{0}-R\right)+\frac{1}{4} k D r \\
& =\frac{1}{4}\left[(\gamma+3)\left(R_{0}-R\right)+k D r\right],
\end{aligned}
$$

using relations (18) and (19).

For $P_{1}=(x, y), \quad P_{2}=(\xi, y), \quad P_{3}=(x, \eta), P_{4}=(\xi, \eta)$, we can write $k \Delta G$ in (17) as follows:

$$
\begin{aligned}
k A G= & k G\left(U^{\prime}\left(P_{1}\right)\right)-k G\left(U^{\prime}\left(P_{2}\right)\right)-k G\left(U^{\prime}\left(P_{3}\right)\right)+k G\left(U^{\prime}\left(P_{4}\right)\right) \\
= & -\left[\frac{1}{4} U^{\prime}\left(P_{1}\right)-\frac{1}{4} U^{\prime}\left(P_{2}\right)-k G\left(U^{\prime}\left(P_{1}\right)\right)+k G\left(U^{\prime}\left(P_{2}\right)\right)\right] \\
& +\left[\frac{1}{4} U^{\prime}\left(P_{3}\right)-\frac{1}{4} U^{\prime}\left(P_{4}\right)-k G\left(U^{\prime}\left(P_{3}\right)\right)+k G\left(U^{\prime}\left(P_{4}\right)\right)\right] \\
& +\frac{1}{4}\left[U^{*}\left(P_{1}\right)-U^{*}\left(P_{2}\right)-U^{*}\left(P_{3}\right)+U^{*}\left(P_{4}\right)\right] \\
& +\frac{1}{4}\left[\bar{U}_{1}\left(P_{1}\right)-\bar{U}_{1}\left(P_{2}\right)-\bar{U}_{1}\left(P_{3}\right)+\bar{U}_{1}\left(P_{4}\right)\right] .
\end{aligned}
$$

The third bracket in (21), namely $\Delta U^{*}$, is zero, since $U^{*}$ is sum of functions of $x$ only and of $y$ only. The fourth bracket in (21) was estimated in $\S 3$, namely

$$
\left|A \bar{U}_{1}\right| \leqq\|I-Q\|_{\infty}|\Lambda(H(I-Q) N(U))| \leqq 4 \cdot \frac{1}{4}(c+C) T^{2}=(c+C) T^{2} .
$$


Let us denote the first and the second brackets in (21) by $\Sigma_{P_{1} P_{2}}$ and $\Sigma_{P_{3} P_{4}}$ respectively. Then by assumption ( 8 ) we have

$$
\begin{aligned}
\left|\Sigma_{P_{1} P_{2}}\right| & \leqq \frac{1}{4} \gamma\left|U^{\prime}\left(P_{1}\right)-U^{\prime}\left(P_{2}\right)\right| \leqq \frac{1}{4} \gamma\left[\left|U^{*}\left(P_{1}\right)-U^{*}\left(P_{2}\right)\right|+\left|\bar{U}_{1}\left(P_{1}\right)-\bar{U}_{1}\left(P_{2}\right)\right|\right] \\
& \leqq \frac{1}{4} \gamma\left[R / A+\frac{1}{2}(c+C) T\right]\left|P_{1}-P_{2}\right| \leqq \frac{1}{2} \gamma T\left[R / A+\frac{1}{2}(c+C) T\right],
\end{aligned}
$$

and an analogous relation holds for $\Sigma_{P_{3} P_{4}}$. Now from (21) and the above argument we derive

$$
|k \Delta G| \leqq \gamma(R / A) T+\frac{1}{2}(c+C) T+0+\frac{1}{4}(c+C) T^{2} .
$$

Finally, from (17), (20), (22) and the assumptions (2), (4) we obtain

$$
\begin{aligned}
\left\|\bar{U}^{*}\right\|_{\infty} \leqq & \frac{1}{4}(\gamma+3)\left(R_{0}-R\right)+\frac{1}{4} k D r+\frac{1}{4} k\left(c+c^{\prime}\right) \\
& +\gamma(R / A) T+\frac{1}{2}(c+C) T+\frac{1}{4}(c+C) T^{2} .
\end{aligned}
$$

Concerning the Lipschitz constant of $\bar{U}^{*}$ we shall need estimates different from those of Case 1. First we have

$$
\begin{aligned}
\bar{U}^{*}(x, y)= & U^{*}(x, y)-k \mathscr{S} Q N U^{\prime}(x, y)=U^{*}(x, y)-k[m(y)+n(x)+\mu] \\
= & -k[m(y)+\mu]+U^{*}(x, y)-k(2 T)^{-1} \int_{0}^{2 T}\left[\Phi(x, \eta)+G\left(U^{\prime}(x, \eta)\right)\right] d \eta \\
= & -k[m(y)+\mu]+\frac{3}{4} U^{*}(x, y)-k(2 T)^{-1} \int_{0}^{2 T} \Phi(x, \eta) d \eta \\
& +\frac{1}{4} \bar{U}_{1}(x, y)+(2 T)^{-1} \int_{0}^{2 T} \frac{1}{4}\left[U^{\prime}(x, y)-U^{\prime}(x, \eta)\right] d \eta \\
& +(2 T)^{-1} \int_{0}^{2 T} \frac{1}{4}\left[U^{\prime}(x, \eta)-k g\left(U^{\prime}(x, \eta)\right)\right] d \eta .
\end{aligned}
$$

For $\bar{U}^{*}(x+p, y)$ we have an analogous expression with $x+p$ replacing $x$. Hence by subtraction we obtain

$$
\begin{aligned}
& \bar{U}^{*}(x+p, y)-\bar{U}^{*}(x, y) \\
& =\left(\frac{3}{4}\right)\left[U^{*}(x+p, y)-U^{*}(x, y]-k(2 T)^{-1} \int_{0}^{2 T}[\Phi(x+p, \eta)-\Phi(x, \eta)] d \eta\right. \\
& \quad+\frac{1}{4}\left[\bar{U}_{1}(x+p, y)-\bar{U}_{1}(x, y)\right] \\
& \quad+(2 T)^{-1} \int_{0}^{2 T} \frac{1}{4}\left[U^{*}(x+p, y)-U^{*}(x, y)-U^{*}(x+p, \eta)+U^{*}(x, \eta)\right] d \eta \\
& \quad+(2 T)^{-1} \int_{0}^{2 T} \frac{1}{4}\left[U^{\prime}(x+p, \eta)-U^{\prime}(x, \eta)-k g\left(U^{\prime}(x+p, \eta)\right)+k g\left(U^{\prime}(x, \eta)\right)\right] d \eta \\
& \quad-(2 T)^{-1} \int_{0}^{2 T} \frac{1}{4}\left[\bar{U}_{1}(x+p, y)-\bar{U}_{1}(x, y)-\bar{U}_{1}(x+p, \eta)+\bar{U}_{1}(x, \eta)\right] d \eta .
\end{aligned}
$$


The third term cancels the first part of the sixth term and the fourth term is zero, since $U^{*}$ is a sum of functions of $x$ only and of $y$ only. Then using (18) and assumptions (5), as shown above, as well as (8), we obtain

$$
\begin{aligned}
\mid \bar{U}^{*}(x+p, y) & -\bar{U}^{*}(x, y) \mid \\
\leqq & {\left[\frac{3}{4}(R / A)+\frac{1}{4} k \lambda\right]|p|+\frac{1}{4} \gamma(2 T)^{-1} \int_{0}^{2 T}\left|U^{\prime}(x+p, \eta)-U^{\prime}(x, \eta)\right| d \eta } \\
& \quad+\frac{1}{2}(c+C) T|p| \\
\leqq & {\left[\left(\frac{3}{4}\right)(R / A)+\frac{1}{4} k \lambda+\frac{1}{4} \gamma\left(R / A+\frac{1}{2}(c+C) T\right)+\frac{1}{2}(c+C) T\right]|p| . }
\end{aligned}
$$

Thus

$$
\operatorname{Lip}_{x} \bar{U}^{*} \leqq \frac{1}{4}[(R / A)(3+\gamma)+k \lambda]+\frac{1}{2}(c+C) T\left(1+\frac{1}{4} \gamma\right)
$$

and the same estimate holds for $\operatorname{Lip}_{y} \bar{U}^{*}$.

Finally, from (23) and (24) we have

$$
\begin{aligned}
\left\|\bar{U}^{*}\right\|= & \left\|\bar{U}^{*}\right\|_{\infty}+A\left\|\bar{U}^{*}\right\|_{*} \leqq \frac{1}{4}(\gamma+3)\left(R_{0}-R\right)+\frac{1}{4} k\left(D r+c+c^{\prime}\right) \\
& +\gamma(R / A) T+\frac{1}{2}(c+C) T+\frac{1}{4}(c+C) T^{2} \\
& +\frac{1}{4} R(3+\gamma)+\frac{1}{4} k \lambda A+\frac{1}{2}(c+C) T\left(1+\frac{1}{4} \gamma\right) A \\
\leqq & \gamma\left(\frac{1}{4}+T / A\right) R_{0}+\frac{3}{4} R_{0}+\frac{1}{4} k\left(D r+c+c^{\prime}+\lambda A\right) \\
& \quad+\frac{1}{2}(c+C) T\left[1+\frac{1}{2} T+\left(1+\frac{1}{4} \gamma\right) A\right],
\end{aligned}
$$

since $0<R \leqq R_{0}$. Therefore $\left\|\left|\overline{U^{*}} \|\right| \leqq R_{0}\right.$ provided (14) holds, as stated above. This shows that $T^{*}: \Omega^{*} \rightarrow \Omega^{*}$ and concludes the proof.

\section{Example}

Let us consider the problem

$$
\begin{gathered}
u_{t t}-u_{\tau \tau}=\phi(t, \tau)+g(u) \\
u(t+T, \tau)=u(t, \tau)=u(t, \tau+T)
\end{gathered}
$$

with $g(u)=-g(-u), g(u)=\arctan u$ for $0 \leqq u<v=\tan (0.95)=1.4$, and $g(u)=0.95+\varrho \sin \sigma(u-\nu)$ for $u \geqq v$, where $\varrho, \sigma$ are arbitrary constants with $|\varrho| \leqq 0.06$ and $|\varrho \sigma| \leqq 0.4$.

Here we have $|g(u)| \leqq 0.95+0.06=1.01$ for all $u$ and we can take $C=$ 1.01. We also have

$$
\begin{gathered}
1 \geqq g^{\prime}(u)=\left(1+u^{2}\right)^{-1} \geqq\left(1+\nu^{2}\right)^{-1}=0.3378 \text { for } 0 \leqq u<v=1.4, \\
\left|g^{\prime}(u)\right|=|\varrho \sigma \cos \sigma(u-\nu)| \leqq|\varrho \sigma| \leqq 0.4 \text { for } u>v
\end{gathered}
$$

Thus, we can take $D=1$. 
For $b=1.2346=\tan (0.89)$ and $b \leqq u<v$ we have $g(u) \geqq 0.89$; for $u \geqq v$ we have $g(u) \geqq 0.95-0.06=0.89$. Thus we can take $B=0.89$.

Again, for $b \leqq u<v$, we have

$$
0.3378=\left(1+\nu^{2}\right)^{-1} \leqq g^{\prime}(u) \leqq\left(1+b^{2}\right)^{-1}=0.3962,
$$

and for the function $g$, which is continuous in $\mathbb{R}$, we have

$$
|g(u)-g(v)| \leqq d|u-v| \text { for all } u, v \geqq b \text { and } d=0.4 \text {. }
$$

Finally, for $k=1$, we have $|u-v-g(u)+g(v)| \leqq \gamma|u-v|$, or

$$
\left|1-(u-v)^{-1}[g(u)-g(v)]\right| \leqq \gamma \text { for all } 0 \leqq u, v \leqq \nu
$$

with $\gamma=1-\left(1+v^{2}\right)^{-1}=0.6622$

For the sake of simplicity we write $R$ for $R_{1}$. If we take

$$
A=1, \quad R_{0}=3, \quad R=1.61, \quad r=0.01, \quad T=0.04
$$

then it remains to verify relations (9)-(14).

Relation (9) becomes $T^{2}(c+C)=(0.0016)(c+1.01) \leqq 0.01$, or

$$
(0.0016) c+0.001616 \leqq 0.01
$$

and this is true for $c \leqq 5.24$.

Relation (10) becomes $R_{0}-R+r=3-1.61+0.01=1.4=\nu$.

Relation (11) becomes

$$
\begin{gathered}
R_{0}-R-2(R / A) T-r=R_{0}-R(1+2 T)-r=3-1.61(1+0.08) \\
-0.01=1.2512>1.2346=b .
\end{gathered}
$$

Relation (12) becomes

$$
\begin{aligned}
c+ & c^{\prime}+\lambda A+2(R / A) d T+(c+C) d T^{2}+d R+2^{-1} d(c+C) T A \\
= & c+c^{\prime}+\lambda+2(1.61)(0.4)(0.04) \\
& +(c+1.01)(0.4)(0.0016)+(0.4)(1.61)+(0.5)(0.4)(c+1.01)(0.04) \\
= & (1+0.00064+0.008) c+c^{\prime}+\lambda+0.05152+0.0006464+0.644+0.00808 \\
\leqq & 0.89=B
\end{aligned}
$$

or, equivalently,

$$
(1.00864) c+c^{\prime}+\lambda+0.70425 \leqq 0.89
$$

and this is true for

$$
\text { (1.00864) } c+c^{\prime}+\lambda \leqq 0.18575
$$


Concerning relation (13), we have $2\left(R_{0}-R\right)=2(3-1.61)=2.78$, whence (13) becomes

$$
\begin{aligned}
k\left[\frac{1}{4}(c+\right. & \left.\left.c^{\prime}+C+\lambda A\right)+\frac{1}{4}(c+C) d T^{2}+\frac{1}{8} d(c+C) T A\right] \\
& +2(R / A) T+\frac{1}{2} k(R / A) d T+\frac{1}{4} k d R \\
= & (0.25)\left(c+c^{\prime}+1.01+\lambda\right)+(0.25)(c+1.01)(0.4)(0.0016) \\
& +(0.125)(0.4)(c+1.01)(0.04) \\
& +2(1.61)(0.04)+(0.5)(1.61)(0.4)(0.04)+(0.25)(0.4)(1.61) \\
= & (0.25+0.00016+0.002) c+(0.25) c^{\prime}+(0.25) \lambda+0.2525+0.0001616 \\
& +0.00202+0.1288+0.01288+0.161 \leqq 2.78=2\left(R_{0}-R\right),
\end{aligned}
$$

in other words,

$$
(0.25216) c+(0.25) c^{\prime}+(0.25) \lambda+0.55736 \leqq 2.78,
$$

and this is true for

$$
(1.00864) c+c^{\prime}+\lambda \leqq 8.89056 .
$$

Concerning relation (14) we have

$$
[1-\gamma-4 \gamma(T / A)] R_{0}=(1-0.6622-0.105952) 3=3(0.231848)=0.695544
$$

and now relation (14) becomes

$$
\begin{aligned}
k(c+ & \left.c^{\prime}+\lambda A+D r\right)+2(c+C) T+(c+C) T^{2}+2(c+C) T A+\frac{1}{2} \gamma(c+C) T A \\
= & c+c^{\prime}+\lambda+0.01+2(c+1.01)(0.04)+(c+1.01)(0.0016) \\
& +2(c+1.01)(0.04)+(0.5)(0.6622)(c+1.01)(0.04) \\
= & (1+0.08+0.0016+0.08+0.013244) c+c^{\prime}+\lambda+0.01+0.0808 \\
& +0.001616+0.0808+0.01337644 \leqq 0.695544
\end{aligned}
$$

and this is true for

$$
(1.17484) c+c^{\prime}+\lambda \leqq 0.50895 \text {. }
$$

Thus we have obtained the relations

$$
\begin{gathered}
c \leqq 5.24 \\
(1.00864) c+c^{\prime}+\lambda \leqq 0.18575 \\
(1.00864) c+c^{\prime}+\lambda \leqq 8.89056 \\
(1.17484) c+c^{\prime}+\lambda \leqq 0.50895
\end{gathered}
$$

We note that the first and the third are consequences of the second one. We conclude that problem (1) has a continuous $T$-periodic (weak) solution provided 
that

$$
\begin{gathered}
|\phi(t, \tau)| \leqq c, \quad|\phi(t, \tau)-\phi(t+\bar{h}+\bar{k}, \tau+\bar{h}-\bar{k})| \leqq 2 \lambda\left(\bar{h}^{2}+\bar{k}^{2}\right)^{\frac{1}{2}}, \\
|\phi(t, \tau)-\phi(t+\bar{h}, \tau+\bar{h})-\phi(t+\bar{k}, \tau-\bar{k})+\phi(t+\bar{h}+\bar{k}, \tau+\bar{h}-\bar{k})| \leqq c^{\prime}, \\
(1.00864) c+c^{\prime}+\lambda \leqq 0.18575, \\
(1.17484) c+c^{\prime}+\lambda \leqq 0.50895 .
\end{gathered}
$$

Since $c^{\prime} \leqq 4 c$ the last two relations are certainly consequences of the following ones:

$$
\text { (5.00864) } c+\lambda \leqq 0.18575, \quad(5.17484) c+\lambda \leqq 0.50895 .
$$

For instance, for

$$
\phi(t, \tau)=10^{-3} \sin (2 \pi / T)(t+\tau), \quad T=0.04,
$$

we have

$$
c=0.001, \quad \phi \in \operatorname{ker} \Xi, \quad c^{\prime}=0
$$

and since

$$
\begin{aligned}
10^{-3} \mid \sin (2 \pi / T)(t+\tau) & -\sin (2 \pi / T)(t+\tau+2 \bar{h}) \mid \\
& \left.=10^{-3} \mid(2 \pi / T) \cos [(2 \pi / T) t+\tau+2 \theta \bar{h})\right]|\cdot| 2 \bar{h}|\leqq 2 \lambda| \bar{h} \mid,
\end{aligned}
$$

we can take

$$
\lambda=(0.001)(2 \pi / T)=0.15708 \text {. }
$$

Thus the inequalities above are certainly satisfied.

Similarly, for

$$
\phi(t, \tau)=10^{-3} \sin (2 \pi / T) t, \quad T=0.04,
$$

we have $c=0.001$ and we can take $c^{\prime}=0.004$. Thus, because

$$
\begin{aligned}
10^{-3} \mid \sin (2 \pi / T) t- & \sin (2 \pi / T)(t+\bar{h}) \mid \\
& =10^{-3}|(2 \pi / T) \cos (2 \pi / T) \cos (2 \pi / T)(t+\theta \bar{h})||\bar{h}| \leqq 2 \lambda|\bar{h}|,
\end{aligned}
$$

we can take

$$
\lambda=(0.5)(0.001)(2 \pi / T)=0.07854 .
$$

Thus the inequalities above are certainly satisfied.

Therefore, both equations

$$
\begin{gathered}
u_{t t}-u_{\tau \tau}=10^{-3} \sin (2 \pi / T)(t+\tau)+g(u), \\
u_{t t}-u_{\tau r}=10^{-3} \sin (2 \pi / T) t+g(u),
\end{gathered}
$$

with $g$ defined as usual in this section, have doubly $T$-periodic solutions satisfying $|u(t, \tau)| \leqq R_{0}+r=3+0.01=3.01$.

Acknowledgement. The first author was supported in part by Project NSF-MCS 8002337 of the National Science Foundation.

The second author was supported partly by the G.N.A.F.A. (Italy) and by the CNR (Italy), in connection with a visit to the University of Michigan, 1980. 


\title{
References
}

1. L. CeSARI, Existence in the large of periodic solutions of hyperbolic partial differential equations. Archive Rational Mech. Anal. 20, 170-190 (1965).

2. L. CESARI, A boundary value problem for quasi linear hyperbolic systems in bicharacteristic canonic form. Ann. Scuola Normale Sup. Pisa (4) 1, 311-358 (1974).

3. L. CESARI, Functional Analysis, nonlinear differential equations, and the alternative method. Nonlinear Functional Analysis and Differential Equations (L. Cesari, R. Kannan, J. D. Schuur, eds.), 1-197, New York; M. Dekker, 1976.

4. L. Cesari \& R. Kannan, Periodic solutions of nonlinear wave equations. Archive Rational Mech. Anal., 82, 295-312 (1983).

5. J. K. HALE, Periodic solutions of a class of hyperbolic equations. Archive Rational Mech. Anal. 23, 380-398 (1967).

6. J. K. Hale, Applications of Alternative Problems. Lecture Notes, Brown University, 1971, 1-69.

7. P. PuCCI, Problemi ai limiti per sistemi di equazioni iperboliche. Boll. Un. Mat. Ital. 16-B, 87-99 (1979).

\author{
Department of Mathematics \\ University of Michigan \\ Ann Arbor \\ and \\ Istituto di Matematica \\ Universitá degli Studi \\ Perugia
}

(Received August 15, 1982) 